\title{
Too much of a good thing: hedonistic homoeostatic dysregulation and other behavioural consequences of excessive dopamine replacement therapy in Parkinson's disease
}

\author{
John D O'Sullivan (id 1,2
}

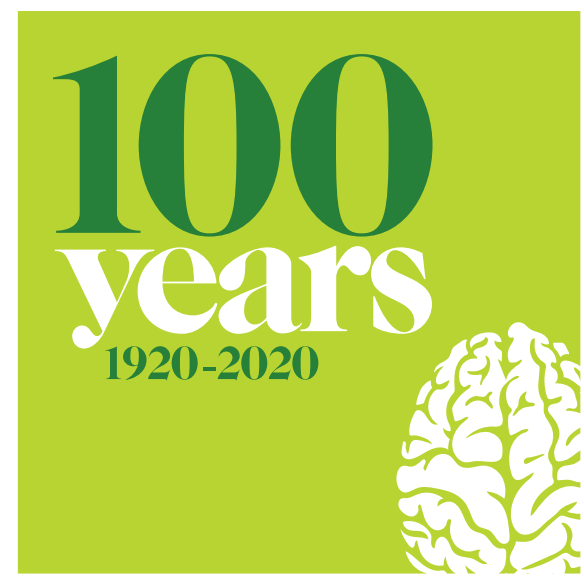

The publication twenty years ago describing excessive therapeutic drug dosing in some Parkinson's disease patients foreshadowed behaviours now well recognised.

The recognition and description of a behavioural syndrome in a group of patients with Parkinson's disease (PD) who take dopamine replacement therapy in quantities beyond what is required to treat motor symptoms 20 years ago ${ }^{1}$ sparked interest for a number of reasons. In addition to its deficiency being integral to PD, dopamine had long been regarded as a critical neurotransmitter involved in reward systems reduction in dopamine associated with depression and increased dopamine with addiction. Within the addiction field, these patients represented a real-life experiment to support the prevailing models suggesting maladaptive mesolimbic dopamine circuits underlying addiction. ${ }^{2}$ Conversely in the field of neurology, the paper highlighted and built on occasional observations and case reports of abnormal behaviour with

${ }^{1}$ Centre for Clinical Research, University of Queensland, Brisbane, Queensland, Australia

${ }^{2}$ Neurology, Royal Brisbane and Women's Hospital, Herston, Queensland, Australia

Correspondence to Dr John D O'Sullivan, Centre for Clinical Research, University of Queensland, Brisbane, QLD 4029, Australia; john.osullivan@uq.edu.au
Too much of a good thing: hedonistic homoeostatic dysregulation and other behavioural consequences of excessive dopamine replacement therapy in Parkinson's disease

Authors: John Daniel O'Sullivan

Year Published: 2000

Number of times cited: 635

excessive use of dopaminergic medication in $\mathrm{PD}$, and proposed a pathophysiological framework within which these behaviours could be understood.

The appellation 'hedonistic homoeostatic dysregulation' was idiosyncratic and served two main purposes in addition to its novelty to neurologists. ${ }^{1}$ First it emphasised the similarity between this behaviour in PD and generic drug abuse or dependence with the term initially used to describe to describe a neurobiological theory of drug addiction. ${ }^{3}$ Second it circumvented the use of the pejorative term 'addiction', particularly when the drugs that are being misused in these patients are necessary to alleviate motor and some non-motor symptoms resulting from pathological degeneration of dopaminergic neurons, at increasing doses with disease progression.

The hedonic homoeostatic dysregulation model of addiction suggests initial anticipation to pleasurable effects of drugs drives increasing drug use, and a cycle of ongoing excessive drug use to avoid the negative affective state induced by drug withdrawal and reflecting a maladaptive reduction in baseline pleasure level. ${ }^{3}$ Others have suggested that neuroadaptations induced in dopamine projections to the nucleus accumbens and related circuitry result in sensitisation to the rewarding effect of the drug and leading to drug 'wanting' or craving rather than drug liking. ${ }^{45}$ This incentive sensitisation model has been supported by evidence of enhanced levodopa-induced dopamine release from the ventral striatum on positron emission tomography (PET) imaging in these patients, ${ }^{6}$ and the clinical observations that craving, drug seeking and hoarding are more common in these patients than deriving pleasure from the dopamine replacement therapy. ${ }^{4}$ These alternative theories have led to a more general term 'dopamine dysregulation syndrome' to describe this behaviour. The syndrome is incorporated in the revised Movement Disorders Society Unified PD Rating Scale (MDS-UPDRS), ${ }^{7}$ and patients with features of the syndrome are often simply labelled as 'dysregulators'.

We reported that patients only feeling appropriately medicated or 'on' when they have drug-induced dyskinesia that can be severe is a clue to hedonistic homoeostatic dyskinesia, and this feature has been observed in subsequent reports. Early disease onset has remained a consistent risk factor in larger series, however, male gender, past or family history of alcohol or other substance abuse or if psychiatric illness have been less reliable risk factors. ${ }^{89}$

The prevalence of hedonistic homoeostatic dysregulation in PD was low, even in our tertiary and quaternary referral centre. A similarly low prevalence was reported from an Italian group ${ }^{10}$ and less than 100 cases were found in the literature in a recent systematic review. ${ }^{9}$ Despite the low occurrence of the syndrome, interest in this paper was generated by our early description of a number of associated behavioural disturbances that are now recognised more commonly PD patients without all the features of hedonistic homoeostatic dysregulation.

Hypersexuality associated with levodopa had been long recognised ${ }^{11}$ but remarkably pathological gambling and shopping had not been well recognised prior to our report. These and other similar appetitive behaviours have become collectively referred to as impulse control disorders (ICDs) and are increasingly recognised, particularly with the use of 
dopamine agonist therapy. ${ }^{12}$ Recent longitudinal studies reveal a high prevalence of ICD with up to $50 \%$ of PD patients who have used dopamine agonists over 5 years. $^{13}$ Punding, originally described in cocaine and amphetamine addicts, involves complex prolonged, purposeless and stereotyped behaviour. Punding had been barely reported in PD prior to our publication ${ }^{14}{ }^{15}$ but was subsequently reported in $14 \%$ of patients with PD taking higher dose of dopamine replacement therapy ${ }^{16}$ and is now well recognised and considered as an ICD-related conditions. Walkabout and drug hoarding in PD first described in our paper are now also considered as ICD-related conditions. The negative affective state we described in these patients after attempted withdrawal of dopamine replacement therapies including dysphoria, depression, irritability and anxiety is now recognised in the dopamine agonist withdrawal syndrome (DAWS). ${ }^{17}$ DAWS has been reported in up to $19 \%$ of patients with PD during taper of dopamine agonist drugs, often complicating management of ICD. ${ }^{18}$

It was perhaps ambitious to describe, define and provide management guidelines for this syndrome in a single publication. The inclusion of diagnostic criteria and practical clinical strategies, however, enhanced the publications appeal. The provisional diagnostic criteria we proposed have generally been replicated in subsequent publications. We highlighted some of the difficulties in applying criteria designed for drug or substance abuse and dependence in these patients. The tolerance to, 'withdrawal' from, and escalating long-term use of dopamine replacement therapies in most patients with advancing PD would fulfil criteria for substance dependence in the Diagnostic and Statistical Manual of Mental Disorders DSM-IV and now for substance use disorder in DSM-V, emphasising the difficulty in using these criteria for this population. ${ }^{19} 20$

The management guidelines we suggested were largely based on our experience in managing the patients described in the paper, however, even 20 years later, no interventions to manage dopamine dysregulation syndrome were considered to have sufficient evidence of efficacy in a recent evidence-based medicine review of non-motor features of PD. ${ }^{21}$ The difficulty we described in attempting to reduce levodopa and dopamine replacement therapy in this population has been shared by others, with drug hoarding and obtaining additional supplies from different providers or now online particularly problematic. Many patients are resistant to therapeutic interventions with only $40 \%-53 \%$ of patients achieving remission in longitudinal studies. ${ }^{822}$

We reported continuous rather than intermittent apomorphine infusion as a therapeutic strategy, though a recent longitudinal study following a cohort of patients who fulfilled the criteria proposed in our original paper found levodopa intestinal gel infusion (LCIG) and subthalamic nucleus deep brain stimulation (DBS) beneficial in a large proportion of their patients. ${ }^{8}$ These device-assisted therapies were not widely available at the time of our report. Patients with dopamine dysregulation undergoing any device assisted therapies still require extensive multidisciplinary intervention and symptoms may persist or even originate after DBS and LCIG.

The increased appreciation and understanding of behavioural consequences of dopamine replacement therapy in PD has its origin in their observation and recognition in clinical cohorts, akin to our initial understanding of the clinical syndrome stemming from James Parkinson's seminal observations a century ago. ${ }^{23}$ This reinforces the roles of comprehensive history taking, caregiver reports and considering non-motor as well as motor responses to the broadening therapeutic interventions in PD and related conditions.

Funding The authors have not declared a specific grant for this research from any funding agency in the public, commercial or not-for-profit sectors.

\section{Competing interests None declared.}

Patient consent for publication Not required.

Provenance and peer review Commissioned; internally peer reviewed.

(c) Author(s) (or their employer(s)) 2020. No commercial re-use. See rights and permissions. Published by BMJ.

\section{A) Check for updates}

To cite O'Sullivan JD. J Neurol Neurosurg Psychiatry 2020;91:566-567.

Received 28 January 2020

Accepted 3 February 2020

Published Online First 5 March 2020

J Neurol Neurosurg Psychiatry 2020;91:566-567. doi:10.1136/jnnp-2019-322378

\section{ORCID iD}

John D O'Sullivan http://orcid.org/0000-0003-04228590

\section{REFERENCES}

1 Giovannoni G, O'Sullivan JD, Turner K, et al. Hedonistic homeostatic dysregulation in patients with Parkinson's disease on dopamine replacement therapies. J Neurol Neurosurg Psychiatry 2000;68:423-8.

2 Ambermoon P, Carter A, Hall W, et al. Compulsive use of dopamine replacement therapy: a model for stimulant drug addiction? Addiction 2012;107:241-7.

3 Koob GF, Le Moal M. Drug abuse: hedonic homeostatic dysregulation. Science 1997;278:52-8.

4 Lawrence AD, Evans AH, Lees AJ. Compulsive use of dopamine replacement therapy in Parkinson's disease: reward systems gone awry? Lancet Neurol 2003:2:595-604.

5 Robinson TE, Berridge KC. The neural basis of drug craving: an incentive-sensitization theory of addiction. Brain Res Brain Res Rev 1993;18:247-91.

6 Evans $A H$, Pavese N, Lawrence AD, et al. Compulsive drug use linked to sensitized ventral striatal dopamine transmission. Ann Neurol 2006:59:852-8.

7 Goetz CG, Tilley BC, Shaftman SR, et al. Movement disorder Society-sponsored revision of the unified Parkinson's disease rating scale (MDS-UPDRS): scale presentation and Clinimetric testing results. Mov Disord 2008;23:2129-70.

8 Cilia R, Siri C, Canesi M, et al. Dopamine dysregulation syndrome in Parkinson's disease: from clinical and neuropsychological characterisation to management and long-term outcome. J Neurol Neurosurg Psychiatry 2014;85:311-8

9 Warren N, O'Gorman C, Lehn A, et al. Dopamine dysregulation syndrome in Parkinson's disease: a systematic review of published cases. J Neurol Neurosurg Psychiatry 2017;88:1060-4.

10 Pezzella FR, Colosimo C, Vanacore N, et al. Prevalence and clinical features of hedonistic homeostatic dysregulation in Parkinson's disease. Mov Disord 2005:20:77-81.

11 Quinn NP, Toone B, Lang AE, et al. Dopa dosedependent sexual deviation. Br J Psychiatry 1983;142:296-8

12 Weintraub $D$, Siderowf $A D$, Potenza MN, et al. Association of dopamine agonist use with impulse control disorders in Parkinson disease. Arch Neurol 2006:63:969-73

13 Corvol J-C, Artaud F, Cormier-Dequaire F, et al. Longitudinal analysis of impulse control disorders in Parkinson disease. Neurology 2018;91:e189-201.

14 Fernandez HH, Friedman JH. Punding on L-dopa. Mov Disord 1999; 14:836-8.

15 Friedman JH, levodopa Pon. Punding on levodopa. Biol Psychiatry 1994;36:350-1.

16 Evans AH, Katzenschlager R, Paviour D, et al. Punding in Parkinson's disease: its relation to the dopamine dysregulation syndrome. Mov Disord 2004:19:397-405.

17 Rabinak CA, Nirenberg MJ. Dopamine agonist withdrawal syndrome in Parkinson disease. Arch Neurol 2010;67:58-63.

18 Yu XX, Fernandez HH. Dopamine agonist withdrawal syndrome: a comprehensive review. J Neurol Sci 2017:374:53-5

19 Bearn J, Evans A, Kelleher M, et al. Recognition of a dopamine replacement therapy dependence syndrome in Parkinson's disease: a pilot study. Drug Alcohol Depend 2004;76:305-10.

20 Hasin DS, O'Brien CP, Auriacombe M, et al. DSM-5 criteria for substance use disorders: recommendations and rationale. Am J Psychiatry 2013;170:834-51.

21 Seppi K, Ray Chaudhuri K, Coelho M, et al. Update on treatments for nonmotor symptoms of Parkinson's disease-an evidence-based medicine review. Mov Disord 2019;34:180-98.

22 Barbosa P, Djamshidian A, Lees AJ, et al. The outcome of dopamine dysregulation syndrome in Parkinson's disease: a retrospective postmortem study. Mov Disord Clin Pract 2018;5:519-22.

23 Parkinson J. An essay on the shaking palsy. London: Sherwood, Neely and Jones, 2002: 223-36. 